\title{
Efecto de los extraíbles en tres propiedades físicas de la madera de Enterolobium cyclocarpum procedente de Michoacán, México
}

\author{
Effect of extractives on three physical properties in Enterolobium \\ cyclocarpum from Michoacan, Mexico
}

\author{
Luz Elena Ávila a*, Marco Antonio Herrera a \\ *Autor de correspondencia: ${ }^{a}$ Universidad Michoacana de San Nicolás de Hidalgo, Facultad de Ingeniería en Tecnología de la \\ Madera, apartado postal 580, CP 58030, Morelia, México, tel./fax: +52-443-3260379, lavila@umich.mx
}

\begin{abstract}
SUMMARY
The presence of extractives in wood has an important effect on its properties and its transformation processes. The aim of this work was to study the effect of extractives on dimensional variation, density and the fiber saturation point of Parota heartwood (Enterolobium cyclocarpum). The behavior of non-extracted specimens was compared to that presented by specimens extracted with acetone and ethanol-water (1:1). The extraction with acetone was performed using soxhlet equipment during effective times of 120 hours of reflux and 260 hours submerged in the solvent; the extraction with ethanol-water (1:1) was achieved in 155 and 370 hours respectively. It was found that extractives give less dimensional variation to Parota hardwood, because they produce a "bulking" effect in the cellular wall which reduces the shrinkage and swelling of wood. This effect was statistically significant in radial, tangential and volumetric determinations; while in longitudinal measurements, this effect was not significant. Density was not affected by the removal of extractives in Parota heartwood. The presence of extractives caused a reduction in the fiber saturation point, this was proved using Stamm's and Babiak and Kúdela's equations.
\end{abstract}

Key words: density, shrinkage, swelling, fiber saturation point, extractives.

\section{RESUMEN}

La presencia de extraíbles en la madera tiene un efecto importante en sus propiedades y en sus procesos de transformación. El objetivo de este trabajo fue evaluar el efecto de los extraíbles en tres propiedades físicas: la variación dimensional, la densidad y el punto de saturación de la fibra de la madera de duramen de parota (Enterolobium cyclocarpum). Se comparó el comportamiento de probetas no extraídas y extraídas con acetona y con etanol-agua (1:1). La extracción con acetona se realizó con equipos Soxhlet durante 120 horas efectivas de reflujo y 260 horas sumergidas en el solvente; y la extracción con etanol-agua (1:1) se realizó con tiempos de 155 y 370 horas, respectivamente. Se encontró que los extraíbles otorgaron menor variación dimensional al duramen de parota al ejercer un efecto de "abultamiento" en la pared celular que disminuyó la contracción y el hinchamiento. Este efecto fue estadísticamente significativo en las determinaciones radiales, tangenciales y volumétricas; mientras que en determinaciones longitudinales no fue significativo. Las densidades anhidra, saturada y básica no se vieron afectadas por la remoción de extraíbles en la madera de parota. La presencia de extraíbles ocasionó una disminución en el punto de saturación de la fibra, lo cual se comprobó empleando las ecuaciones de Stamm y de Babiak y Kúdela.

Palabras clave: densidad, contracción, hinchamiento, punto de saturación de la fibra, extraíbles.

\section{INTRODUCCIÓN}

Los extractivos son un grupo de compuestos químicos de la madera que se pueden extraer con solventes polares y no polares. Estos componentes, también llamados materiales extraños, se encuentran en los lúmenes de las células, en los intersticios de la pared celular o en las cavidades intercelulares. Constituyen 4 al $10 \%$ del peso anhidro de madera normal de especies que crecen en climas templados y en más del $20 \%$ en madera de especies tropicales (Rowell 1984). La presencia de extraíbles no modifica la estructura de la madera y contribuye en un bajo porcentaje a su masa, sin embargo, tiene un gran efecto en sus propiedades y en sus procesos de transformación (Poblete et al. 1991). La generalidad de los autores coincide en señalar que la presencia de mayores cantidades de extraíbles depositados tanto en la pared celular como en los lúmenes, ocasionan un incremento en la densidad de la madera (e.g. Tsoumis 1991).

Por otro lado, la alta cantidad de extraíbles reduce la contracción e hinchamiento (Adamopoulos 2002, Hernández 2007bc); la reducción es proporcional al espacio ocu- 
pado por los extraíbles en las paredes celulares (fenómeno de abultamiento), espacio que hubiera ocupado un volumen igual de agua en las fibras (Tsoumis 1991).

El punto de saturación de la fibra puede variar considerablemente dependiendo del tipo de sustancias extractivas presentes, del tipo de solvente que se usó para la extracción y de la estructura misma de la madera; en general, la presencia de los extraíbles disminuye el punto de saturación de la fibra (Popper et al. 2006, Hernández 2007a).

El objetivo de este trabajo es evaluar el efecto de los extraíbles en la variación dimensional, la densidad y el punto de saturación de la fibra de la madera de duramen de parota (Enterolobium cyclocarpum (Jacq.) Griseb). La especie estudiada se eligió debido a su alto contenido de extraíbles (de 24,5 a 32,4\% con base en el peso anhidro de la madera, Ochoa et al.1995, Ramos et al. 2011) condición que permite esperar que los extraíbles ocasionen una menor variación dimensional y punto de saturación de la fibra, así como una mayor densidad.

\section{MÉTODOS}

El material experimental consistió en madera de duramen de E. cyclocarpum. El árbol fue colectado en la localidad de "El Copalito", municipio de Tacámbaro, Michoacán, México (figura 1). De una troza de $110 \mathrm{~cm}$ de largo con un diámetro total de $32 \mathrm{~cm}$ y diámetro del duramen de $19 \mathrm{~cm}$, se obtuvieron dos discos de $10 \mathrm{~cm}$ de espesor. La cercanía en la ubicación de las probetas provenientes de un solo ejemplar, permitió mayor uniformidad de los contenidos de extraíbles, condición que admitió estudiar el efecto del tratamiento y minimizar el efecto de la variabilidad de la especie (Ramos et al. 2011). Del primer disco se prepararon 12 probetas en forma de prismas rectangulares de 20 x 20 x $30 \mathrm{~mm}$ y orientadas en las direcciones radial, tangencial y longitudinal del plano leñoso para destinarlas

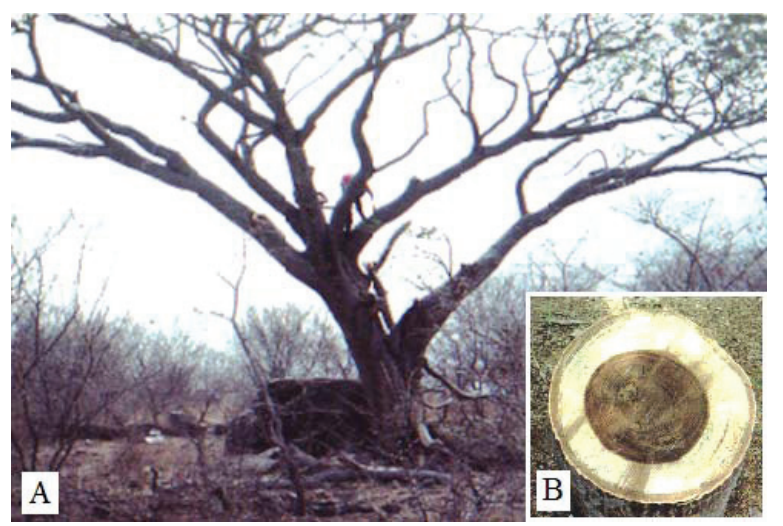

Figura 1. E. cyclocarpum. A) Ejemplar de estudio. B) Disco para obtención de material de estudio.

Enterolobium cyclocarpum. A) Specimen of study. B) Slice to obtain study materials. a extracción (probetas extraídas). El segundo disco se selló y se mantuvo en refrigeración hasta la conclusión de la preparación de las probetas extraídas, momento en que se prepararon 21 probetas como testigo (probetas no extraídas). Todas las probetas estuvieron libres de nudos, grietas, desviación de la fibra y de ataque de hongos e insectos.

Preparación de probetas extraídas. Las probetas se mantuvieron en reflujo en equipos Soxhlet. En la primera fase de extracción se utilizó acetona, las probetas se mantuvieron por 120 horas efectivas bajo reflujo y 260 horas sumergidas en el solvente. Los reflujos se efectuaron con una velocidad de 4 a 5 por hora y una temperatura de rocío de $50{ }^{\circ} \mathrm{C}$. Para la segunda fase de extracción se utilizó una mezcla de etanol:Tagua (1:1) con un periodo efectivo de extracción de 155 horas y 370 horas sumergidas en el solvente. La temperatura de rocío de la mezcla etanol:[agua varió entre 60 y $64^{\circ} \mathrm{C}$.

Proceso de desorción y adsorción. La estrategia experimental consistió en observar el proceso de desorción de la madera a partir de su condición saturada (contenido de humedad $>30 \%$ ) hasta su condición anhidra (contenido de humedad $=0 \%$ ) y del proceso inverso de adsorción. El proceso de desorción se realizó en dos etapas. La primera etapa consistió en un acondicionamiento durante 481 horas, a una temperatura de $23{ }^{\circ} \mathrm{C}$ y una humedad relativa de $82 \%$; en la segunda etapa, las probetas se secaron a 103 ${ }^{\circ} \mathrm{C}$ hasta su condición anhidra. Las mediciones fueron realizadas en las tres direcciones de anisotropía de la madera utilizando un micrómetro con precisión de $\pm 0,01 \mathrm{~mm}$; el peso se registró empleando una balanza con precisión de $\pm 0,01 \mathrm{~g}$; y el volumen se determinó por el método de inmersión en mercurio, en apego a la Norma D 2395-93, Método D, Modo IV (ASTM 2000). Las lecturas en la primer etapa se realizaron cada 2 horas, las primeras 24 horas del proceso; con incrementos de una hora cada 24 horas, hasta realizar lecturas cada 8 horas, lapso que se mantuvo constante hasta el término de esta etapa. En la segunda etapa se registraron las lecturas hasta alcanzar peso constante.

Para el proceso de adsorción las probetas fueron colocadas en inmersión en agua destilada a temperatura ambiente hasta su saturación. El registro de las lecturas en esta etapa se realizó en intervalos de tiempo como se indicó para la primera etapa de desorción.

Contenido de humedad. El contenido de humedad actual de la madera se determinó por el método de diferencia de pesos: el contenido de humedad correspondiente a cada estado de humedad de la madera se calculó a partir del peso de la probeta registrado en cada determinación, en relación al peso de la madera medido en la condición anhidra de cada probeta [1].

$\mathrm{CH}_{\mathrm{a}}=\frac{\mathrm{P}_{\mathrm{a}}-\mathrm{P}_{0}}{\mathrm{P}_{0}}[100]$ 
donde:

$\mathrm{CH}_{\mathrm{a}}=$ contenido de humedad actual

$\mathrm{P}_{\mathrm{a}}=$ peso en condición de humedad actual

$\mathrm{P}_{0}=$ peso en condición anhidra

Variación dimensional. La contracción en las direcciones radial, tangencial, longitudinal y volumétrica se calculó empleando la ecuación [2].

$$
\beta=\frac{D_{\mathrm{i}}-\mathrm{D}_{\text {Сна }}}{\mathrm{D}_{\mathrm{i}}}[100]
$$

Donde:

$\beta=$ contracción al contenido de humedad actual de la madera

$\mathrm{D}_{\mathrm{i}}=$ dimensión en condición saturada

$\mathrm{D}_{\mathrm{CHa}}=$ dimensión al contenido de humedad actual de la madera

El hinchamiento radial, tangencial, longitudinal y volumétrico se determinó de acuerdo a la ecuación [3].

$\alpha=\frac{\mathrm{D}_{\mathrm{CH}}-\mathrm{D}_{0}}{\mathrm{D}_{0}}[100]$

Donde:

$\alpha=$ hinchamiento al contenido de humedad actual de la madera

$\mathrm{D}_{0}=$ dimensión en condición anhidra

$\mathrm{D}_{\text {Сна }}=$ dimensión al contenido de humedad actual de la madera

Densidad. La densidad se calculó a partir de las mediciones de peso y volumen registradas en la condición anhidra y la etapa de adsorción. La densidad anhidra, densidad saturada y densidad básica en probetas no extraídas y extraídas se calcularon con las ecuaciones [4], [5] y [6].

$\rho_{0}=\frac{\mathrm{P}_{0}}{\mathrm{~V}_{0}}$

Donde:

$\rho_{0}=$ densidad anhidra

$\mathrm{P}_{0}=$ peso en condición anhidra

$\mathrm{V}_{0}=$ volumen en condición anhidra

$\rho_{\mathrm{s}}=\frac{\mathrm{P}_{\mathrm{s}}}{\mathrm{V}_{\mathrm{s}}}$

Donde:

$\rho_{\mathrm{s}}=$ densidad saturada

$\mathrm{P}_{\mathrm{s}}=$ peso en condición saturada

$\mathrm{V}_{\mathrm{s}}^{\mathrm{s}}=$ volumen en condición saturada
$\rho_{\mathrm{b}}=\frac{\mathrm{P}_{0}}{\mathrm{~V}_{\mathrm{s}}}$

Donde:

$\rho_{\mathrm{b}}=$ densidad básica

$\mathrm{P}_{0}=$ peso en condición anhidra

$\mathrm{V}_{\mathrm{s}}=$ volumen en condición saturada

Punto de saturación de la fibra. Para la determinación del punto de saturación de la fibra se utilizaron las ecuaciones [7] y [8].

$\operatorname{PSF}_{\mathrm{BK}}=\left[\frac{1}{\rho_{\mathrm{b}}}-\frac{1}{\rho_{0}}\right] \rho_{\text {нго }} \times 100$

Donde:

$\mathrm{PSF}_{\mathrm{BK}}=$ punto de saturación de la fibra de acuerdo con Babiak y Kúdela (1995)

$\rho_{\mathrm{b}}=$ densidad básica

$\rho_{0}=$ densidad anhidra

$\rho_{\mathrm{H} 2 \mathrm{O}}=$ densidad del agua

$\mathrm{PSF}_{\mathrm{S}}=\frac{\beta_{\mathrm{v}}}{\rho_{\mathrm{b}}}$

Donde:

$\mathrm{PSF}_{\mathrm{S}}=$ punto de saturación de la fibra de acuerdo con Stamm (1964)

$\beta_{\mathrm{v}}=$ contracción volumétrica

$\rho_{\mathrm{b}}=$ densidad básica

Análisis estadísticos. Para la determinación del efecto de los extraíbles en la variación dimensional (contracción e hinchamiento), la densidad y el punto de saturación de la fibra; los datos fueron sometidos al análisis de varianza con un factor. El valor de probabilidad alfa establecido para calificar como significativas a las diferencias encontradas con la fuente de variación fue de 0,05 . Las determinaciones estadísticas se realizaron con el software Statistica 7.0.

\section{RESULTADOS}

Contracción. Las probetas extraídas presentaron valores mayores: $170,7 \%$ en contracción radial $(P<0,001)$, $165,3 \%$ en contracción tangencial $(P<0,001)$ y $116,8 \%$ en contracción volumétrica $(P<0,001)$, la diferencia en contracción longitudinal no fue significativa $(P=0,9820)$ (cuadro[1). Se presentó colapso en algunas probetas extraídas después de 10 horas de iniciada la etapa de desorción, después de 14 horas el $75 \%$ de ellas lo presentaban. Las probetas no extraídas no presentaron muestras visibles de colapso. 
Cuadro 1. Propiedades físicas en probetas no extraídas (PNE) y probetas extraídas (PE) de madera de duramen de E. cyclocarpum.

Physical properties in non-extracted samples (NES) and extracted samples (ES) of heartwood of Enterolobium cyclocarpum.

\begin{tabular}{|c|c|c|c|}
\hline Propiedad & PNE & $\mathrm{PE}$ & $P$ \\
\hline \multicolumn{4}{|c|}{ Contracción } \\
\hline$\beta_{\mathrm{r}}$ & 1,9019 & 5,1488 & $<0,001^{* * *}$ \\
\hline$\beta_{\mathrm{t}}$ & 3,8799 & 10,2920 & $<0,001^{* * *}$ \\
\hline$\beta_{1}$ & 0,6112 & 0,5399 & $0,9820 \mathrm{~ns}$ \\
\hline$\beta_{\mathrm{v}}$ & 6,4435 & 13,9690 & $<0,001^{* * *}$ \\
\hline \multicolumn{4}{|c|}{ Hinchamiento } \\
\hline$\alpha_{\mathrm{r}}$ & 3,1385 & 6,9162 & $<0,001^{* * *}$ \\
\hline$\alpha_{t}$ & 6,0823 & 15,0320 & $<0,001^{* * *}$ \\
\hline$\alpha_{1}$ & 0,6701 & 0,6785 & $0,9544 \mathrm{~ns}$ \\
\hline$\alpha_{\mathrm{v}}$ & 10,5829 & 26,9393 & $<0,001^{* * *}$ \\
\hline \multicolumn{4}{|c|}{ Densidad } \\
\hline$\rho_{0}$ & 0,42 & 0,43 & $0,3867 \mathrm{~ns}$ \\
\hline$\rho_{\mathrm{s}}$ & 0,96 & 0,92 & $0,1088 \mathrm{~ns}$ \\
\hline$\rho_{\mathrm{b}}$ & 0,38 & 0,36 & 0,4646 ns \\
\hline \multicolumn{4}{|c|}{ Punto de saturación de la fibra } \\
\hline $\mathrm{PSF}_{\mathrm{BK}}$ & 17,63 & 47,75 & $<0,001^{* * *}$ \\
\hline $\mathrm{PSF}_{\mathrm{S}}$ & 17,30 & 52,39 & $<0,001^{* * *}$ \\
\hline
\end{tabular}

$\beta$ : contracción (\%), $\alpha$ : hinchamiento (\%); los subíndices $r, t, 1, v$ corresponden a radial, tangencial, longitudinal y volumétrico, respectivamente. $\rho$ : densidad $\left(\mathrm{g} \mathrm{cm}^{-3}\right)$; los subíndices 0 , s y b corresponden a anhidra, saturada y básica. PSF $_{\mathrm{BK}}$ : punto de saturación de la fibra de acuerdo a Babiak y Kúdela (\%). PSF : punto de saturación de la fibra de acuerdo a Stamm (\%). $P$ : probabilidades límites en ANDEVA ***: $P<0,001$; ns: $P>0,05$.

Hinchamiento. Se obtuvieron incrementos de $120,4 \%$ en hinchamiento radial $(P<0,001), 147,1 \%$ en hinchamiento tangencial $(P<0,001)$ y de $154,6 \%$ en hinchamiento volumétrico $(P<0,001)$ en probetas extraídas respecto a las probetas no extraídas. La diferencia del hinchamiento longitudinal entre probetas extraídas y no extraídas no fue significativa $(P=0,9544)$. En la figura 2 se observa que las probetas no extraídas alcanzaron la estabilización dimensional en las direcciones radial $(P<0,001)$ y tangencial $(P<0,01)$ con mayor rapidez que las probetas extraídas, al presentar contenidos de humedad menores. Para la dirección longitudinal esta variación no fue significativa $(P=0,1954)$.

Densidad. La densidad en estado anhidro $\left(\rho_{\mathrm{o}}=\mathrm{P}_{\mathrm{o}} \mathrm{V}_{\mathrm{o}}^{-1}\right)$, la densidad en estado saturado $\left(\rho_{\mathrm{s}}=\mathrm{P}_{\mathrm{s}} \mathrm{V}_{\mathrm{s}}^{-1}\right)$ y la densidad básica $\left(\rho_{\mathrm{b}}=\mathrm{P}_{\mathrm{o}} \mathrm{V}_{\mathrm{v}}^{-1}\right)$ no presentaron diferencias significativas entre probetas no extraídas y extraídas $(P=0,3867$, $P=0,1088$ y $P=0,4646$ respectivamente) (cuadro 1 ).

Punto de saturación de la fibra. El punto de saturación de la fibra en probetas extraídas fue mayor que en probetas

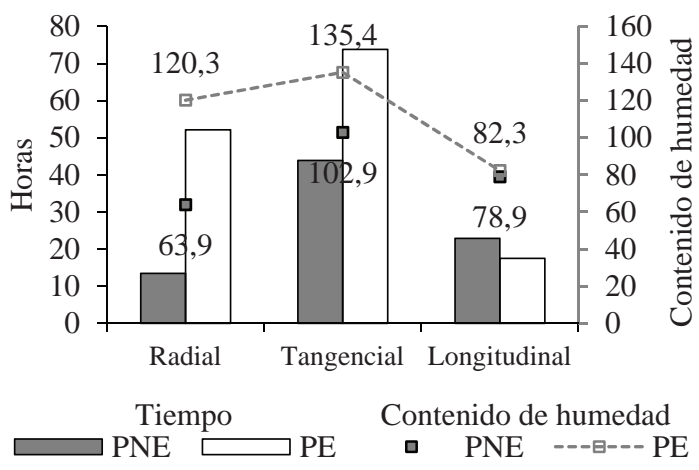

Figura 2. Tiempos (en horas) y contenidos de humedad (en porcentaje) de estabilización dimensional durante la etapa de adsorción. Se indican las direcciones de los ejes anisotrópicos en probetas no extraídas (PNE) y probetas extraídas (PE) de madera de duramen de E. cyclocarpum.

Dimensional stabilization times (in hours) and moisture content (in percentage \%) during the adsorption phase. They indicate the directions of the anisotropic axes in non-extracted samples (NES) and extracted samples (ES) of heartwood of Enterolobium cyclocarpum.

no extraídas por los dos métodos de cálculo $(P<0,001)$ (cuadro 1).

\section{DISCUSIÓN}

Contracción. El comportamiento de contracción e hinchamiento de la especie en estudio, puede deberse a la variación del contenido de extraíbles, de su tipo y de su localización; lo que provoca una diferencia en la difusión del agua en la madera. Esto explica la mayor variabilidad en las probetas extraídas, ya que el proceso de extracción puede provocar una relocalización de los extraíbles, y por lo tanto una mayor variabilidad en la difusión. El incremento de la contracción radial, tangencial y volumétrica en probetas extraídas fue evidente, duplicando los valores con respecto a las probetas no extraídas. Choong y Achmadi (1991) reportan resultados similares para especies tropicales de la India, Chen et al. (1995) para encino (Quercus sp.) y secuoya (Sequoia sempervirens (D. Don) Endl.) y Adamapoulos (2002) para Robinia pseudoacacia L. Sólo Taylor (1974) reporta una disminución en la contracción radial en probetas extraídas en varias especies de coníferas y latifoliadas.

Por otro lado, el colapso de las probetas extraídas pudo deberse a que algunos extraíbles actúan como plastificantes a altas temperaturas, los que pueden ablandar la pared celular. El colapso puede ocurrir por el incremento de la contracción y el debilitamiento de la pared celular durante la pérdida de agua libre aparente. Las probetas no extraídas no mostraron evidencia de colapso, porque la acción de incremento de volumen de los extraíbles y su oclusión en el lumen puede controlar o prevenir el colapso (Choong y Achmadi 1991). Por su parte, Chafe (1987) 
propone la teoría que explica que la concentración de extraíbles presentes en las paredes celulares puede ser tan alta en algunas especies, que su acción de "abultamiento o relleno" limita severamente la contracción y previene o minimiza el colapso de un material que de otro modo podría ser propenso a colapsar.

Hinchamiento. El contenido de humedad máximo y el tiempo en que alcanzan la estabilización las probetas extraídas fueron mayores que en probetas no extraídas. Este fenómeno puede deberse a que los espacios ocupados en un inicio por los extraíbles, fueron remplazados por agua, incrementando el contenido de humedad y el tiempo necesario para su adsorción. Torelli et al. (2006) constatan que el alto porcentaje de extraíbles y la cantidad total de fenoles solubles en madera de Abies alba Mill son altamente significativos para la disminución del contenido de humedad en equilibrio en duramen y menor en albura. Por su parte, Hernández (2007b) determina que el contenido de humedad en equilibrio y la variabilidad dimensional disminuyen cuando el contenido de extraíbles solubles en acetona se incrementa. El efecto de los extraíbles en el hinchamiento radial y tangencial observado en este trabajo coincide, en forma general, con los obtenidos por Hernández (2007c) quien establece que los extraíbles solubles en acetona y metanol, posiblemente situados dentro de las paredes celulares, afectan negativamente el hinchamiento radial y tangencial, respectivamente.

Densidad. Los resultados obtenidos en este trabajo se oponen a lo reportado por Tsoumis (1991) que indica que la densidad se ve afectada positivamente (en menor o mayor grado) por la presencia de grandes cantidades de extraíbles. Por el contrario, Ona et al. $(1997,1998)$ determinan que los extraíbles afectan negativamente la densidad básica en base a la madera libre de extraíbles de Eucalyptus globulus Labil. y E. camaldulensis Dehn.

Punto de saturación de la fibra. La alta significancia estadística en los resultados obtenidos sugiere que las maderas con altos contenidos de extraíbles poseen un punto de saturación de la fibra menor que las que no los tienen, en este sentido Popper et al. (2006) observan una alta correlación entre el punto de saturación de la fibra con los extraíbles solubles en etanol-toluol y Hernández (2007a) con los extraíbles solubles en acetona. La función de los extraíbles en este caso, es ocupar espacios en la pared celular, que pudieran ser sustituidos por agua, ocasionando la saturación de la pared con una menor cantidad de este líquido, lo que provoca un punto de saturación de la fibra menor.

\section{CONCLUSIONES}

Los extraíbles presentes en la madera de duramen de parota le otorgan menor variación dimensional al ejercer un efecto de "abultamiento" en la pared celular que dismi- nuye la contracción y el hinchamiento radial, tangencial y volumétrico. El punto de saturación de la fibra es afectado negativamente por la presencia de los extraíbles, ya que éstos ocupan espacios en la pared celular, que pueden ser sustituidos por agua. Contrario a lo esperado, los extraíbles no tienen efecto significativo en la variación dimensional en la dirección longitudinal ni en la densidad.

\section{REFERENCIAS}

Adamapoulos S. 2002. Influence of hot-water extractives on radial and tangential shrinkage of black locust wood (Robinia pseudoacacia L.). Holz als Roh- und Werkstoff 60: 377-378.

ASTM (American Society for Testing and Materials, US). 2000. Standard test methods for specific gravity of wood and wood-based materials. D 2395-93. Annual book of ASTM standards. Section four: Construction. Volume 04.10: Wood. West Conshohocken, PA, USA. pp. 361-368.

Babiak M, J Kúdela. 1995. A contribution to the definition of the fiber saturation point. Wood Science and Technology 29(1): 217-226.

Chafe SC. 1987. Collapse, volumetric shrinkage, specific gravity and extractives in Eucalyptus and other species. Part 2: The influence of wood extractives. Wood Science and Technology 2(1): 27-41.

Chen Y, ET Choong, HM Barnes. 1995. Effect of selected watersoluble bulking chemicals on moisture diffusion and dimensional stability of wood. Forest Products Journal 45(5): 84-90.

Choong ET, SS Achmadi. 1991. Effect of extractives on moisture sorption and shrinkage in tropical woods. Wood and Fiber Science 23(2): 185-196.

Hernández RE. 2007a. Effects of extraneous substances, wood density and interlocked grain on fiber saturation point of hardwoods. Wood Material Science and Engineering 2(1): 45-53.

Hernández RE. 2007b. Moisture sorption properties of hardwoods as affected by their extraneous substances, wood density, and interlocked grain. Wood and Fiber Science 39(1): 132-145.

Hernández RE. 2007c. Swelling properties of hardwoods as affected by their extraneous substances, wood density, and interlocked grain. Wood and Fiber Science 39(1): 146-158.

Higuchi T. 1997. Biochemistry and molecular biology of wood. Springer. Berlín, Germany. 362 p.

Hillis WE. 1986. Forever Amber. A story of the secondary wood components. Wood Science and Technology 20: 203-227.

Ochoa HG, LR Bravo, M Enriquez, MG Lomelí. 1995. Aislamiento y caracterización de extractos maderables. Avances de Ingeniería Química 5(2): 161-165.

Ona T, T Sonoda, K Ito, M Shibata. 1997. Relationship between various extracted basic densities and wood chemical components in Eucalyptus camaldulensis. Wood Science and Technology 31: 205-216.

Ona T, T Sonoda, K Ito, M Shibata. 1998. Relations between various extracted basic densities and wood chemical components in Eucalyptus globulus. Journal Wood Science 44: 165-168.

Poblete H, S Rodríguez, M Zárate. 1991. Extraíbles de la made- 
ra sus características y efectos sobre la utilización de esta materia prima. Publicación docente No. 34. Valdivia, Chile. Facultad de Ciencias Forestales. Universidad Austral de Chile. 51 p.

Popper R, P Niemz, M Torres. 2006. Einfluss des Extraktstoffanteils ausgewählter fremdländischer Holzarten auf deren Gleichgewichtsfeuchte. European Journal of Wood and Wood Products 64(6): 491-496.

Ramos D, L Ávila, H Ochoa, J Rutiaga. 2011. Contenido de sustancias extraíbles en la madera de Enterolobium cyclocarpum (Jacq.) Griseb. (Leguminosae). Ciencia Nicolaita 53: 7-19.

Rowell R. 1984. The chemistry of solid wood. Washington D. C.,
USA. American Chemical Society. 614 p.

Stamm AJ. 1964. Wood and cellulose science. New York, USA. Ronald Press. 549 p.

Taylor FW. 1974. Effect of extraction on the volume dimension and specific gravity of solid wood blocks. Wood Science 6(4): 346-404.

Torelli N, J Trajkoviü, V Serti. 2006. Influence of phenolic compounds in heartwood of Silver fir (Abies alba Mill.) on the equilibrium moisture content. Holz als Roh- und Werkstoff 64: 341-342.

Tsoumis G. 1991. Science and technology of wood. New York, USA. Van Nostrand Reinhold. 494 p.

Recibido: 29.08.11

Aceptado: 27.06.12 